\title{
Psychiatry and the WWW: some implications
}

\author{
C. Senior, M. L. Phillips and A. S. David
}

This paper highlights the role that the World Wide Web (KWWW has to play as an aid to psychiatiry. A basic history of the WWW is provided as is an introduction to some search techniques involved with the WWW. The itterature on applications potentially relevant to poychiatry is reviowed using computer search facilities (BIDS, Psychlit and Mediline). The WWW is one of the aspects of the Internet that possesses a huge potential for exploltation, both the clinical and research poychiatrist are able to benefit from its use.

The evolution of the Internet from its conception in the early sixties to its present day existence is an impressive technological achievement. The original raison d'être, a military telecommunications network, has now developed into an efficient and powerful forum for business (Kazuaki, 1990; Janal, 1995), scientific (Thomas, 1996) and medical correspondence (Bergman, 1994). The
Internet, a global communications network, has as its essential components for use, a computer, a device for translating the computer signal to one compatible with a telephone line (called a modem') and a telephone line. With these an individual can gain access to a vast amount of information. The structure of the Internet is complex: it has many levels (Huang \& Allessi, 1996) through which people can communicate (see Fig. 1).

It is necessary, however, to convey the importance of each level to the user. For example, electronic mail (e-mail) and the World Wide Web (WWW) are grouped together in the same layer in the Huang \& Allesi model, yet their difference can be demonstrated by considering the results of two separate surveys. The first was an e-mail based survey and it recorded a total of 12 responses out of a possible 400 (Tse et al. 1995). The second survey was implemented

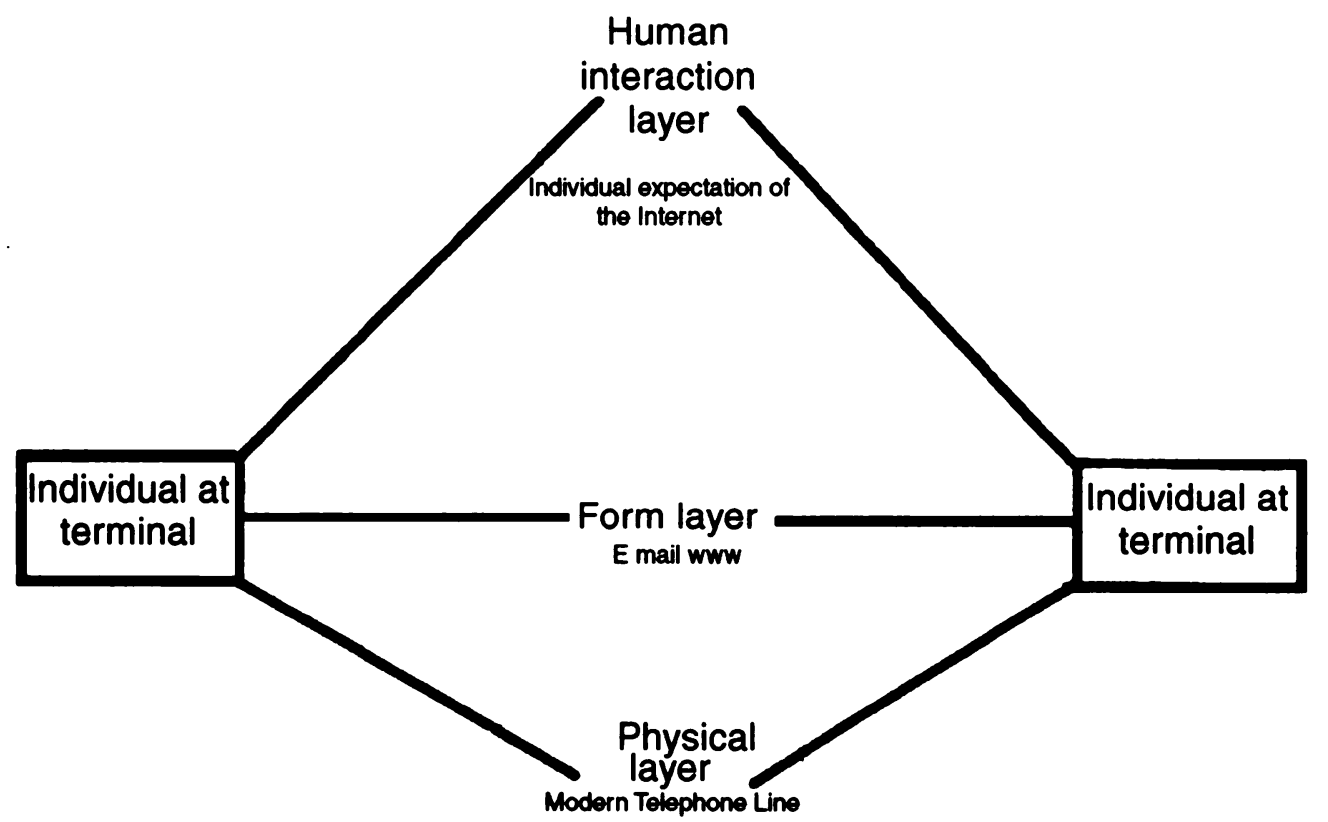

Figure 1. A schematic summany of the Huang \& Alessi (1996) model showing the different layers of the Internet. 
through the WWW and the recorded response rate for this survey was eighteen thousand out of a potential subject pool of millions (Pitkow \& Recker, 1996).

The implications of the Internet for psychiatry are many and varied, (see Huang \& Allessi, 1996 for a review). This paper will focus on the use of the WWW since a majority of the Internet users have experience of this and it is also the current method for downloading a range of media.

The WWW is a 'front end' of the Internet, that is the component with which the user has initial interaction. It was created by Tim Berners-Lee at the European Laboratory for Particle Physics (CERN) in order to ensure a rapid flow of information in a user-friendly manner (BernersLee et al, 1994). The WWW is accessed through web browsers. These browsers are pieces of software code that allow the user to download and view text, pictures, sounds or even movie clips, examples of these are Netscape 2.2 and Microsoft's Explorer. Individuals are able to gain access to the large amount of information on the Internet through a computer language called HyperText Markup Language (HTML). There are limitations to HTML's ability to do certain things. These limitations are now minimised by the advent of the new computer languages, Java and Java-script. Java was developed by the computer manufacturers Sun Microsystems and can be used to write applets (small applications) for creating enhanced features on a web browser. An example of the use of Java can be seen with the Georgia Institute of Technology's WWW user surveys. In this instance Java was used to structure a complex on-line questionnaire with adaptive questions that were aimed at improving subsequent questions on the value of previous statements (Kehoe \& Pitkow, 1996) (<www.cc.gatech.edu/ gvu/user-surveys/papers $>$ ).

\section{How difficult is it to use the WWW?}

In order to construct a web site (defined as a suite of hypertext documents on the Internet) all that is required is a rudimentary knowledge of HTML. Many texts offer an alternative to learning HTML simply constructing a document via a word processor, for example a curriculum vitae or research proposal, and then inserting the HTML clauses or 'Tags' (HTML Tags are simple clauses used to mark text to be displayed as formatted text in a WWW Browser) (Ford, 1995; Lemay, 1995). Using this procedure a basic understanding of HTML can be achieved. Once this is done the systems administrator will ensure that the information is placed on the Internet. Most institutes will restrict access to the WWW files to a few individuals, in most cases the computer systems administrator, purely for security reasons (Thomas, 1996).

At first glance the Internet does seem to be foreboding but there are certain tools that help in the navigation of the WWW. The best known of these tools is the search engine. Out of all of the search engines 'Yahoo' is the most well known. Typing the address of the Yahoo search engine (www.yahoo.com) in to the location window of your browser takes one to the Yahoo home page where you are prompted for a search string i.e. a brief label or heading. A search engine like Yahoo will direct an individual to another location which holds information similar to the initial search string. If specific information about a particular topic is required, like the names and addresses of other clinicians who have encountered a specific condition in their duties, an information engine is needed. An Information engine like 'The Human Search' site (www.mind spring.com/ cjoh/humansearch/) will find any information about a particular topic and, if the results are negative, direct the user to a further source of information.

\section{How can the WWW help the health professional?}

During the course of routine patient care, from the initial interview to the design and implementation of the package of care, many decisions have to be made. The WWW could potentially be implemented at every stage. Leeuw et al (1995) compared the data quality of the various forms of computer assisted interviewing techniques (CAI) and maintained that CAI techniques do provide the surveyor with a higher data quality content than traditional paper and pen interviews. The question of the balance between objectivity and subjectivity and of the level of intrusion that the computer terminal may produce in the interview room has to be considered. Clearly the clinician's opinion is relevant in the initial diagnosis of the patient, but a trainee psychiatrist should be expert in seeking the opinion of others. A database of stereotypical verbatim remarks by patients and their implications would be beneficial to the trainee psychiatrist (see appendix for a list of examples of relevant databases).

Previous research has demonstrated the validity of the Internet as an international database (Groves, 1996; Welch \& Krantz, 1996) although it should be remembered that most developing countries do not have access to viable telecommunications and are therefore outside the remit of the WWW. This is a situation that has been addressed by Bernard Lown, an American clinician who developed 'SatelLife', a low cost communications satellite that enables medical professionals in developing countries to commu- 
nicate with colleagues in the more developed world (see Groves, 1996 for a review of Lown's project).

The gradual advance of technology, while generally accepted in some circles, is met with scepticism in others. LaPorte \& Hibbitt's (1996) article in the British Medical Journal noted that the lack of control that individuals have over their published work may be one reason to do away with the traditional form of the academic journal and to turn to the Internet as a more contemporary form of publication. LaPorte \& Hibbert's article was answered by a number of journal editors in the same issue. Although the authors of these articles made many robust statements in defence of the traditional journal they made no point as to why the Internet should not be used as a forum for the publication of scientific information. The ease of publication and the size of the readership would ensure that. very soon, up to date information could be readily available to all practising psychiatrists. The problem would be the uncertainty over the quality of this information that would be placed on the WWW.

\section{Can the WWW contribute to research?}

The WWW is ideally suited to playing a role in research. Hypertext allows a range of media to be accommodated on one page and this has facilitated a range of WWW experiments. Welch \& Krantz (1996) used audio-sound files to create a suite of psychoacoustical experiments and attempted to demonstrate the validity of the WWW as a research tool. Although the Welch \& Krantz (1996) paper obtained a significant number of hits to the site (i.e. people participating via the $\mathrm{WWW}$ ) the number of viable responses to the experiments were too low. A further study carried out on the WWW which compared the results of laboratory and on-line subject samples on the determinants of female attractiveness found that there was significant convergence between the laboratory-based data sets and the WWW data sets, thereby providing support for the utility of the WWW as a research tool (Krantz et al, 1997). The main difficulty with WWWbased research is quantifying the selection biases inherent in subject populations recruited in this way and the 'denominator' or total population from which such subjects are drawn. Clearly further work in this area is warranted and indeed the first major longitudinal investigation of the demographic variables of the WWW population shows a gradual shift away from the people described as 'technology developers' to more 'early adapters/seekers of information'. This shift away from the technological developers is also reflected in the trends of the other variables that were investigated. For example, over the five-year study, samples taken every year indicate that the level of users with doctoral degrees is gradually falling (decline of $24 \%$ over the test period). The gender of the users is also showing a trend towards a more equal representation of the sexes (Kehoe \& Pitkow, 1996). Thus we are beginning to see a more representative sample of the population using the Internet.

The concept of a representative sample can be taken further when one considers the fact that individuals in a WWW survey, due to its anonymity, may be less prone to a social desirability effect in answering questions about themselves. Unfortunately, the fact that the surveyor is not seen by the surveyed allows anybody that fills in a WWW questionnaire to adopt an identity, gender and even a personality that they see fit. The WWW also provides a medium for complex social interactions. Turkle (1996) refers to an increase in the incidence of malicious encounters in conference groups on the WWW that have lead to criminal proceedings. The phrase 'virtual rape' was coined by Dibbell (1993) (cited in Turkle, 1996) and has since been used to describe aggressive, non-consensual encounters on areas of the Internet by both Turkle (1996) and Bruckman (1994) ("Approaches to managing deviant behaviour in virtual communities" on-line document <FTP:// FTP.media.mit.edu/pub/asb/papers/deviancechi-94>).

One of the many areas that the WWW could contribute to is the dissemination of information. A traditional journal article can take many months between submission and publication, while an article published on the WWW can take a few seconds to appear in its final format although, as noted earlier, this raises questions as to the validity of on-line articles. The British Psychology Society has attempted to address this situation by creating a dual on-line and printed system where the user can read and provide a commentary on submitted articles before they are published. The edited on-line commentary is published along side the printed article and the learned journal project can be found at <journals. eecs.qub.ac.uk>

\section{Conclusions}

The Internet is a communications phenomenon that enables individuals and companies to communicate on equal terms. It effectively removes the barriers of social class, race and gender and ensures that the point of view of the individual can be heard. During its relatively short existence, the WWW has grown exponentially and has now become an omnipotent medium, that has effectively reached the furthest 
outskirts of the Internet. At present its main uses are easy access to information, 'on the job' browsing, and as a population research tool.

\section{Acknowledgements}

We thank Dr S. Arcuri for many helpful discussions over the content of this paper. We would also like to thank the anonymous reviewer who provided us with several helpful comments with regards to the first draft. C. S. is partially funded by the John Mackintosh Trust, Gibraltar.

\section{Appendix}

1. Focus Home Health

<http://www.focushealth.com/>.

Primarily concerned with psychiatric home health.

2. The Institute of Psychiatry

<http://www.iop.bpmf.ac.uk>.

This site contains many links to other general psychiatric-based Internet sites.

3. American Psychiatric Press

<http://www.appi.org/>.

Contains links to on-line journals and periodicals. 4. Psychguides

<http://www.psychguides.com/>.

Detailed guides on the treatment of a variety of psychiatric disorders.

5. Pilots

<http://www.dartmouth.edu/dms/ptsd/index. html $>$.

A database organised by the national centre for post-traumatic stress disorders in the USA.

6. The New England Journal of Medicine <http://www.nejm.org/>.

The Internet version of the journal.

7. University Hospitals Cleveland Health Files

<http:www.uhhs.com/toheal/hfiles/>.

General introduction to a number of conditions.

8. The schizophrenia home page

<http://www.schizophrenia.com/>.

Up-to-date collection on aspects pertaining to schizophrenia.

9. Obsessive-compulsive disorders

<http://www.fairlite.com/ocd/>.

10. Medscape

<http://www.medscape.com>.

On-line resource for patient care.

\section{References}

BERGMAN, R. (1994) The world at their fingertips: rural providers turn to the Internet: rural health professionals use e-mail based telemedicine. Hospital and Health Networker, 68(14), 52.
Berners-Lee. T., Cailuau, R., Luotonen, A., et al (1994) The World Wide Web. Communications of the ACM. 37(8), 76-82.

FORD. A. (1995) Spinning The Web: How to provide Information on the Internet. Hove: International Thompson Publishing.

GROVES, T. (1996) SatelLife: getting relevant information to the developing world. British Medical Journal, 313. 1606-1609.

HuAng, M. \& Alessi, N. (1996) The Internet and the Future of Psychiatry. American Joumal of Psychiatry. 153. 861-869.

Janal, D. (1995) On-line Marketing Handbook: How to Sell Advertise. Publicise and Promote your Products and Services on the Internet and Commercial On-line systems. New York: Von Nostrand Reinhold.

KAZUAKI, K. (1990) Recent developments and future trends in marketing research in Japan using new electronic media. Journal of Advertising Research, 24, 53-57.

KenOE, C. \& PrTKOW, J. (1996) Surveying the Territory: GVUs Five WWW User Surveys. The World Wide Web Joumal, 1, 60-65.

KRANTZ, J., BALLARD, J. \& SCHER, J. (1997) Comparing the results of laboratory and World Wide Web samples on the determinants of female attractiveness. Behaviour. Research Methods, Instruments and Computers, 29. 264-269.

LAPORTE, R. \& HIBBITTS, B. (1996) Rights, wrongs, and journals in the age of cyberspace. British Medical Journal, 313, 21-28.

LeEuw, E. D., Hox, J. \& SNuKERS, G. (1995) The effect of computer assisted interviewing on data quality: A review. Journal of the Market Research Society. 37. 325-343.

LEMAY, L. (1995) Teach Your Self More Web Publishing with HTML in a Week. New York: Macmillan Computer Publishing.

PTTKOW, J. \& RECKER, M. (1996) Using the Web as a survey tool: Results from the second WWW user survey. Journal of Computer Networks and ISDN Systems, 27. 809-822

THOMAS, B. (1996) The Internet for Scientists and Engineers (2nd edn). Oxford: Oxford University Press.

TSE, A., TSE, K., YIN, C., et al (1995) Comparing two methods of sending out questionnaires: $E$-mail versus mail. Journal of the Market Research Society. 37, 441446.

TURKLE. S. (1996). Life on the Screen: Identity in the Age of the Internet. New York: Weidenfeld and Nicolson.

WELCH, N. \& KRANTZ. J. (1996) The World Wide Web as a medium for psychoacoustical demonstrations and experiments: Experience and results. Behaviour Research Methods, Instruments and Computers, 28. 192-196.

*C. Senior, Research Worker, M. Phillips, Lecturer, and A. S. David, Professor of Cognitive Neuropsychiatry, Section of Neuropsychiatry, Institute of Psychiatry, De Crespigny Park Denmark Hill, London SE5 8AF; e-mail: c.senior@iop.bpmf.ac.uk

"Correspondence 